\title{
Relationship between brain iron deposition and mitochondrial dysfunction in idiopathic Parkinson's disease
}

\author{
Jannik Prasuhn 1,2,3†, Martin Göttlich ${ }^{2,3 \dagger}$, Friederike Gerkan ${ }^{1,2,3}$, Sofia Kourou ${ }^{1,2,3}$, Britt Ebeling 1,2,3, \\ Meike Kasten ${ }^{1,3,4}$, Henrike Hanssen ${ }^{1,2,3}$, Christine Klein ${ }^{1,2}$ and Norbert Brüggemann ${ }^{1,2,3^{*}}$ (D
}

\begin{abstract}
Background: The underlying pathophysiology of Parkinson's disease is complex, involving different molecular pathways, including brain iron deposition and mitochondrial dysfunction. At a molecular level, these disease mechanisms are likely interconnected. Therefore, they offer potential strategies for disease-modifying treatments. We aimed to investigate subcortical brain iron deposition as a potential predictor of the bioenergetic status in patients with idiopathic Parkinson's disease.

Methods: Thirty patients with idiopathic Parkinson's disease underwent multimodal MR imaging (T1, susceptibilityweighted imaging, SWI) and ${ }^{31}$ phosphorus magnetic resonance spectroscopy imaging. SWI contrast-to-noise ratios served as a measure for brain iron deposition in the putamen, caudate, globus pallidus, and thalamus and were used in a multiple linear regression model to predict in-vivo energy metabolite ratios.

Results: Subcortical brain iron deposition, particularly in the putamen and globus pallidus, was highly predictive of the region-specific amount of high-energy-containing phosphorus metabolites in our subjects.

Conclusions: Our study suggests that brain iron deposition but not the variability of individual volumetric measurements are highly predictive of mitochondrial impairment in vivo. These findings offer the opportunity, e.g., by using chelating therapies, to improve mitochondrial bioenergetics in patients with idiopathic Parkinson's disease.
\end{abstract}

Keywords: Parkinson's disease (PD), Mitochondria, Iron

\section{Background}

Various molecular disease mechanisms are associated with nigral and extranigral neurodegeneration in patients with Parkinson's disease (PD), often determining disease onset and progression (Timpka et al. 2017). Two such molecular alterations involve brain iron homeostasis and mitochondrial function disturbances (Muñoz et al. 2016). Although the concept of

\footnotetext{
*Correspondence: norbert.brueggemann@neuro.uni-luebeck.de ${ }^{\dagger} J a n n i k$ Prasuhn and Martin Göttlich contributed equally to this work ${ }^{1}$ Institute of Neurogenetics, University of Lübeck, Ratzeburger Allee 160, 23538 Lübeck, Germany

Full list of author information is available at the end of the article
}

mitochondrial dysfunction involves distinct pathophysiological aspects, e.g. impaired mitophagy and altered mitochondrial dynamics, the final common pathway is bioenergetic depletion (Park et al. 2018). The underlying idea that iron metabolism changes and mitochondrial disturbances are relevant for the disease development refers to the initial, environmental agent-related studies (involving MPTP, 6-OH-DOPA, rotenone, or paraquat), that impair mitochondrial homeostasis (Gaki and Papavassiliou 2014). In-vivo models have furthered our understanding of these environmental agents, revealing increased iron deposition in subcortical brain structures following mitochondrial impairment (Mochizuki original author(s) and the source, provide a link to the Creative Commons licence, and indicate if changes were made. The images or other third party material in this article are included in the article's Creative Commons licence, unless indicated otherwise in a credit line to the material. If material is not included in the article's Creative Commons licence and your intended use is not permitted by statutory regulation or exceeds the permitted use, you will need to obtain permission directly from the copyright holder. To view a copy of this licence, visit http://creativecommons.org/licenses/by/4.0/ 
et al. 1994). Subcortical brain iron deposition in PD has been extensively studied using neuroimaging and postmortem brain examinations (Zhang et al. 2010; Trufanov et al. 2019; Barbosa et al. 2015; Wang et al. 2016). Histopathological investigations have demonstrated that iron deposition is mainly localized to the mitochondria on a subcellular level, stressing the importance of this organelle in regulating intracellular iron metabolism (Muñoz et al. 2016). Mitochondria are responsible for the macromolecular iron integration in metalloproteins, Fe-Sulfur clusters, or heme groups (Liang and Patel 2004; Mena et al. 2011). Previous reports highlighted that increased oxidative stress (e.g., by the inhibition of complex I of the electron transport chain) leads to an impaired assembly of Fe-Sulfur clusters, forcing the mitochondria to import even more iron (Lee et al. 2009). This action might be a self-promoting mechanism as the resulting disproportion of divalent and trivalent iron could increase oxidative stress (Moos and Morgan 2004). Based on these in vitro interactions, the aggravating effects of iron and mitochondrial dyshomeostasis would be reasonable to study for the potential development of disease-modifying treatment strategies (Kakhlon et al. 2010; Sohn et al. 2008; Devos et al. 2020). In this context, specific chelating agents cross the blood-brain barrier, e.g. deferoxamine or deferiprone, might thus rescue iron-overloaded mitochondria by cellular iron redistribution (Kakhlon et al. 2010; Chan et al. 2018). To the best of our knowledge, non-invasive studies combining brain iron deposition (by susceptibility-weighted imaging, SWI) and bioenergetic depletion (by ${ }^{31}$ phosphorus magnetic resonance spectroscopy imaging, ${ }^{31} \mathrm{P}$-MRSI) have not yet been performed in PD. Therefore, our primary hypothesis was to test whether (i) subcortical brain iron deposition or (ii) the individual volumetric measurements are predictive of bioenergetic depletion in patients with PD. The combination of these two imaging modalities might not only help to recapitulate in-vitro and preclinical in-vivo findings to understand disease pathophysiology in human subjects but might also serve as a measure of patient stratification in future clinical trials.

\section{Methods}

\section{Recruitment and clinical assessment}

The present study and all subsequent experimental procedures have been performed in accordance with the revised version of the Declaration of Helsinki. Before the enrollment of the first study participant, this study has been approved by the ethics committee of the University of Lübeck (AZ 18_945). All study participants gave written informed consent before participation in this study. We confirmed the diagnosis of PD following the MDS clinical diagnostic criteria as evaluated by trained movement disorders specialists (JP, HH, NB) (Postuma et al. 2015). The clinical examination included the general patient history and demographic data, potential MRI contraindications, concomitant illnesses, medication including the levodopa-equivalent daily dosage (LEDD), and a standardized clinical assessment following the MDS-UPDRS protocol (Goetz et al. 2008). All patients were regularly taking antiparkinsonian medication, were in the ON state, not fasting, and rested for at least one hour before the start of the imaging procedure.

\section{MRI sequences and analyses}

All MRI measurements were performed at the CBBM Core Facility Magnetic Resonance Imaging on a $3 \mathrm{~T}$ Siemens MAGNETOM Skyra Magnetic Resonance Imaging scanner.

\section{T1 imaging}

We employed a three-dimensional T1-weighted MPRAGE sequence (64-channel head/neck coil) for structural imaging following subsequent imaging parameters: $1 \times 1 \times 1 \mathrm{~mm}^{3}$ voxel size; $192 \times 256 \times 256 \mathrm{~mm}^{3}$ field of view; TR $=1900 \mathrm{~ms}$; TE $=2.44 \mathrm{~ms}$; $\mathrm{TI}=900 \mathrm{~ms}$; flip angle $9^{\circ}$; GRAPPA acceleration factor 2 along anterior/posterior phase-encoding direction, total scan time $4 \mathrm{~min}$ and 33 s). All acquired neuroanatomical images were evaluated by consulting neuroradiologists to preclude relevant brain lesions.

\section{Susceptibility-weighted imaging}

We performed SWI using a standard Siemens 3D highspatial-resolution fully velocity corrected gradient-echo MRI sequence with the following image parameters: voxel size $0.9 \times 0.9 \times 1.5 \mathrm{~mm}^{3} ; 220 \times 220 \times 120 \mathrm{~mm}^{3}$ field of view; $\mathrm{TR}=27 \mathrm{~ms}$; $\mathrm{TE}=20 \mathrm{~ms}$; flip angle $15^{\circ}$; transversal orientation; right/left phase-encoding direction; total scan time $4 \mathrm{~min}$ and $54 \mathrm{~s}$ ).

\section{${ }^{31}$ Phosphorus magnetic resonance spectroscopy imaging}

We used a dual tuned quadrature head coil ${ }^{1} \mathrm{H} /{ }^{31} \mathrm{P}$ (RAPID Biomedical) for $3 \mathrm{~T}$ and applied a 3D Chemical Shift Imaging (CSI) Free Induction Decay sequence (CSIFID) to acquire MRSI data. The protocol parameters were as follows: voxel size $30 \times 30 \times 30 \mathrm{~mm}^{3} ; 240 \times 240 \times 240$ $\mathrm{mm}^{3}$ field of view; $\mathrm{TR}=2000 \mathrm{~ms} ; \mathrm{TE}=2.3 \mathrm{~ms}$; sixfold weighted averaging; flip angle $50^{\circ}$; spectral bandwidth $2000 \mathrm{~Hz}$; vector size 1024; Hamming filtering (width 100\%); Nuclear overhauser effect disabled; WALTZ-4 decoupling, total scan time $8 \mathrm{~min}$ and $4 \mathrm{~s}$. CSI grid placement and the volume of interest covering the basal ganglia are highlighted in Fig. 1. Adjustments and shimming 

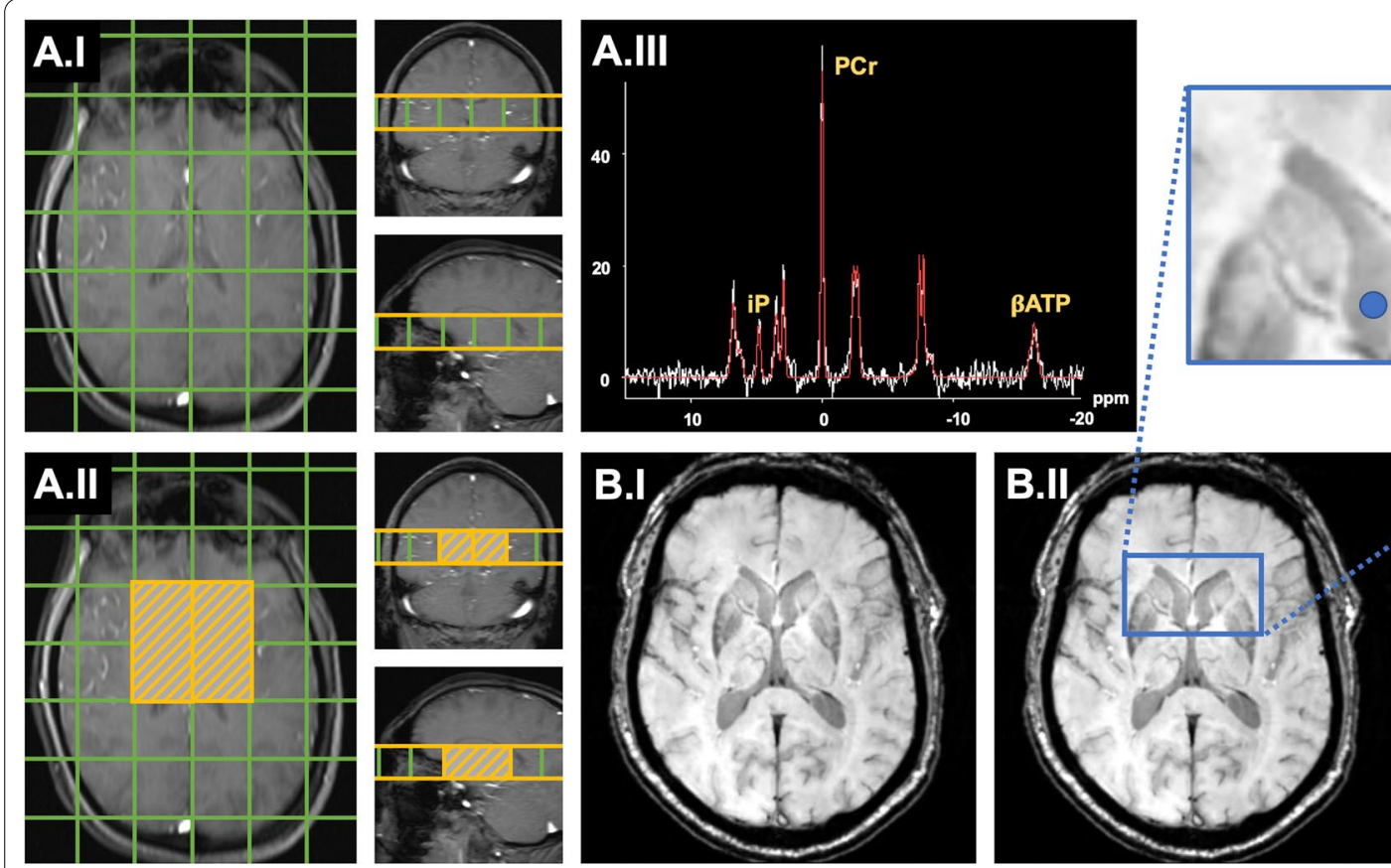

Fig. 1 Methodological approaches for the analysis of the multimodal neuroimaging data. In A, analyses of ${ }^{31}$ P-MRSI measurements are summarized; in B, the approach on calculations of CNRs (as derived from SWI). Panel A.1 illustrates the voxel size and CSI grid placement (green) for ${ }^{31}$ P-MRSI measurements in axial, coronal, and sagittal planes. In Panel A.ll, the voxels of interest (VOIs) for subcortical brain regions are highlighted for each hemisphere (orange hatched). One exemplary ${ }^{31} \mathrm{P}-\mathrm{MRSI}$ spectrum (white line) and the respective model line fit (red line) is shown in Panel A.III. The metabolites of relevance for this study are labeled in yellow. For the sake of readability, other peaks are not marked, as they were not of interest to the hypothesis of this study. In Panel B.I, an exemplary SWI image of one study participant in the axial plane is shown. In Panel B.II, we highlighted the reference ROI placement (blue circles) in the lateral ventricles by a magnified snippet (blue framework). 31 P-MRSI ${ }^{31} \mathrm{Phosphorus}$ magnetic resonance spectroscopy imaging. ATP adenosine triphosphate, CSI chemical shift imaging, iP inorganic phosphate, $P C r$ phosphocreatinine, ppm parts per million, $R O /$ region of interest, SWI susceptibility-weighted imaging, VOI voxel of interest

were performed on a manually selected volume that was slightly larger than the volume of interest.

\section{Neuroimaging analyses}

Volumetric analysis We performed volumetric measurements of subcortical brain structures on $\mathrm{T} 1$ native space images following the segmentation via the wellestablished FIRST suite of the FMRIB software library (v6.0) (Jenkinson 2008; Patenaude et al. 2011). We have chosen the respective options to derive only the left- and right-sided segmentation of the putamen, caudate, globus pallidus, and thalamus. Images of all subjects were manually controlled for segmentation errors before the subsequent statistical analysis. We calculated the subcortical volumes based on the derived segmentations, known voxel size, and voxel number employing standard functions of $f$ slmaths and fslstats (FSLUTILS suite).

Assessment of subcortical brain iron deposition by susceptibility-weighted imaging A measure for brain iron depositions was derived from SWI images. SWI images were linearly coregistered to the native space T1 images of the respective subjects using the FMRIB's Linear Image Registration Tool (Jenkinson and Smith 2001; Jenkinson et al. 2002). For the computation of SWI mean voxel intensities, we used the T1-derived segmentation masks and standard functions of $f$ slmaths and $f$ slstats. To standardize the voxel intensities of subcortical SWI measures, we expressed the mean voxel intensities as the contrast-to-noise ratio (CNR) referenced to a localized CSF signal. Therefore, we created spheres with a diameter of $4 \mathrm{~mm}$ in the MNI space for the lateral ventricles neighboring the subcortical structures of interest (right: $x: 93, y: 132, z: 82$; left: $x: 85$, y: 132, z: 82) by fslmaths (as highlighted for the axial plane in Fig. 1). We non-linearly normalized the native space T1 images of each subject to the MNI152_T1_1mm template using the FMRIB's Non-Linear Image Registration Tool (FNIRT) suite following the recommendations by Andersson et al. (2007). We transferred the CSF spheres from the standard space to the individual native space for each subject using the invwarp function of FNIRT. Correct sphere placement was manually controlled for each subject, and fslmaths and fslstats standard functions computed the mean voxel intensity and the standard deviation (SD) for 
the CSF signal. After data extraction, we calculated the CNR for each subcortical ROI following the equation: $\left(\right.$ mean $_{\mathrm{ROI}}-$ mean $\left._{\mathrm{CSF}}\right) / \mathrm{SD}_{\mathrm{CSF}}$. Here, lower CNR values indicate increased iron deposition, and higher CNR values decreased iron deposition.

Evaluation of the bioenergetic state by ${ }^{31}$ phosphorus magnetic resonance spectroscopy data ${ }^{31} \mathrm{P}-\mathrm{MRSI}$ spectra were fitted in the time domain using the well-established AMARES (advanced method for accurate, robust, and efficient spectral fitting) algorithm (Vanhamme et al. 1997) as implemented in the Oxford Spectroscopy Analysis toolbox (Purvis et al. 2017) (OXSA; https://github.com/oxsat oolbox/oxsa) for Matlab ${ }^{\circledR}$. A 1st-order phase correction to compensate for receiver dead-time was performed before spectral fitting. The AMARES fitting algorithm was developed for the evaluation of MRSI spectra. The algorithm allows imposing prior knowledge and boundary conditions on the fitting parameters, i.e. chemical shift, phase, amplitude and line width for each metabolite, to constrain the nonlinear least-squares fit. A Gaussian line-shape for each peak was used. The following metabolites were taken into account: Adenosine triphosphate (ATP), phosphocreatine (PCr), inorganic phosphate (iP), phophocholine (PC), phosphoethanolamine (PE), glycerophosphocholine (GPC), glycerophosphoethanolamine (GPE), diphosphoglycerate (DPG) and nicotinamide adenindinucleotide (NAD). Initial values for the peak positions, i.e., chemical shifts relative to $\mathrm{PCr}(0.0 \mathrm{ppm})$, of the phosphorous metabolites were taken from Ren et al. (2015). Due to homonuclear ${ }^{31} \mathrm{P}_{-}{ }^{31} \mathrm{P}$ J-coupling, the $\alpha$ - and $\gamma$ ATP signals are split into duplets with the same amplitude, and the $\beta A T P$ signal is split into a 1-2-1 triplet. J-coupling is taken into account and imposed as prior knowledge. The J-coupling constant was constrained to $16 \mathrm{~Hz}$ (Jung et al. 1997). All initial values, prior knowledge and boundary conditions are summarized in Additional file 1: Table S1. After fitting the spectra, we determined the area under each signal and calculated metabolite ratios $((\beta \mathrm{ATP}+\mathrm{PCr}) /$ iP, $\beta A T P / i P$, and $\mathrm{PCr} / \mathrm{iP}$ ) for each of the four voxels of interest (VOI) to account for the high degree of withinspectra autocorrelation of metabolites and to standardize the potentially differing alimentary intake of phosphoruscontaining nutrients. Based on the intra-individual differing rostral brain length and resulting imprecise localization of distinct subcortical brain structures within the CSI grid, rostrally neighboring (but hemispherically different) VOIs were averaged for subsequent analyses.

\section{Statistical analysis}

We computed all statistical analyses using GraphPad Prism (version 9.0.0 88) on a MacOS Mojave (version
10.14.6) workstation. Demographics and clinical characteristics are reported as mean \pm SD. Initially, we analyzed whether hemispherical side differences for distinct subcortical voxels (for ${ }^{31} \mathrm{P}$-MRSI) or neuroanatomical structures (SWI CNR or volumetric measures) were present using paired sample t-tests. As the presence of hemispherical side differences may foster our findings' interpretability, these analyses were exploratory and are subsequently reported as uncorrected p-values.

We computed six multiple linear regression models to test our predefined hypotheses:

The three metabolite ratios served as the dependent variables, the CNR values, or the volumetric measures of the four selected subcortical brain structures served as the independent variables. These results were corrected for multiple comparisons $(n=6)$ applying the Bonferroni correction, resulting in an adjusted significance level $\left(\mathrm{P}_{\text {adj }}\right)$ of $\mathrm{P}_{\mathrm{adj}} \leq 0.0083$. For significant findings on an uncorrected statistical level, we also calculated parameter estimates and the goodness of fit for our multiple regression models to enhance our results' interpretability. In addition, we used a combination of graphical and numerical diagnostics to test the validity of prior assumptions for multiple regression models (absence of multicollinearity, normality of residuals, and the presence of homoscedasticity), which are highlighted in Fig. 2, Additional file 1: Figure S2, Table S2 and Table 1 . To explore potential relationships between clinical parameters and PD-related brain changes, we performed correlation analyses for demographic and clinical data with our neuroimaging derived parameters. We performed logistic regression analyses for dichotomous variables (i.e., sex and the more-affected side) and Pearson's correlations for age, disease duration, MDS-UPDRS subscores, Hoehn and Yahr scale, and the LEDD (Schade et al. 2020) with our neuroimaging markers. The exploratory Pearson's correlation analyses were illustrated via a heatmap (consisting of correlation coefficients). To decrease the number of statistical tests and for the sake of enhanced interpretability, we averaged over both hemispheres.

\section{Results}

Thirty right-handed patients with PD were enrolled of whom 19 (63.3\%) were male and eleven (36.7\%) female with a mean age of $62.5 \pm 9.4$ years and a disease duration of $6.9 \pm 5.0$ years. The disease severity is characterized by MDS-UPDRS-I (7.3 \pm 4.1$)$, MDS-UPDRS-II (8.8 \pm 6.8$)$, MDS-UPDRS-III ( $24.5 \pm 13.2)$, MDS-UPDRSIV $(5.1 \pm 3.3)$ scores, and a Hoehn and Yahr Stage of $2.1 \pm 0.8$. Our study subjects took a levodopa equivalent 

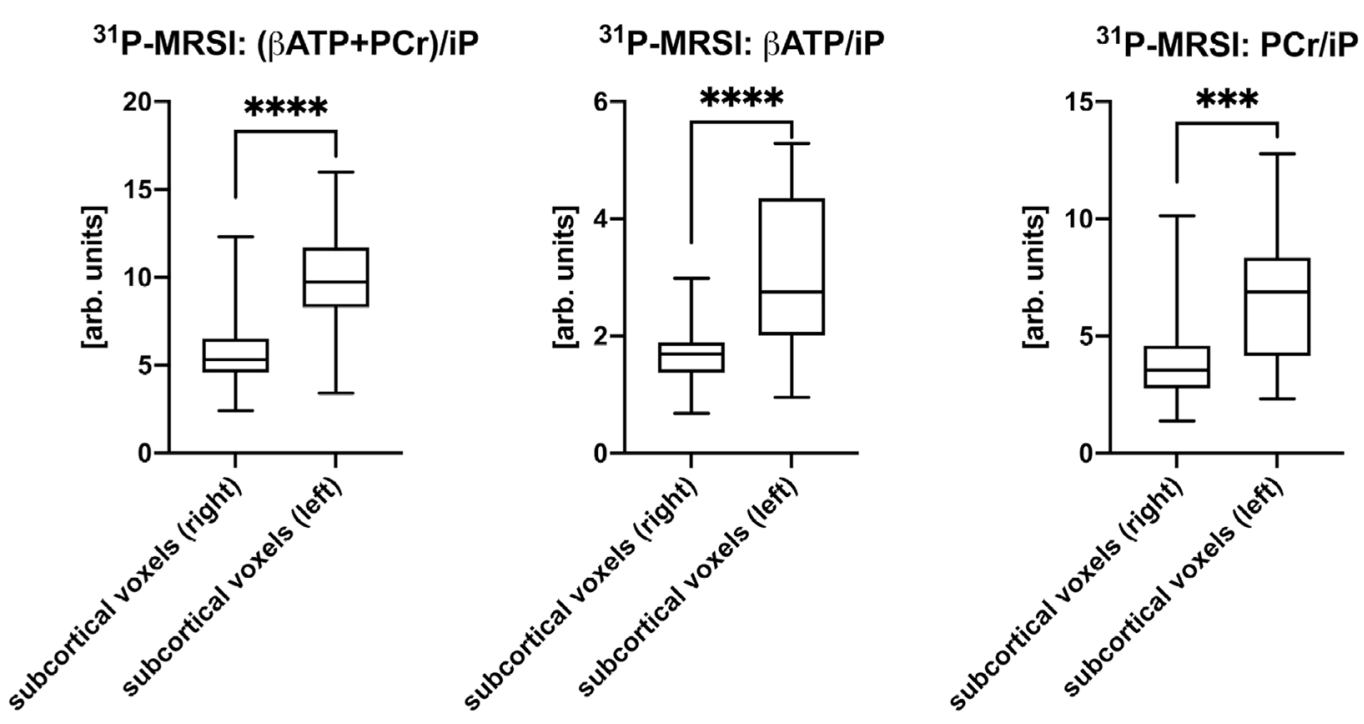

\section{Normalized Intensities (SWI) of subcortical nuclei}
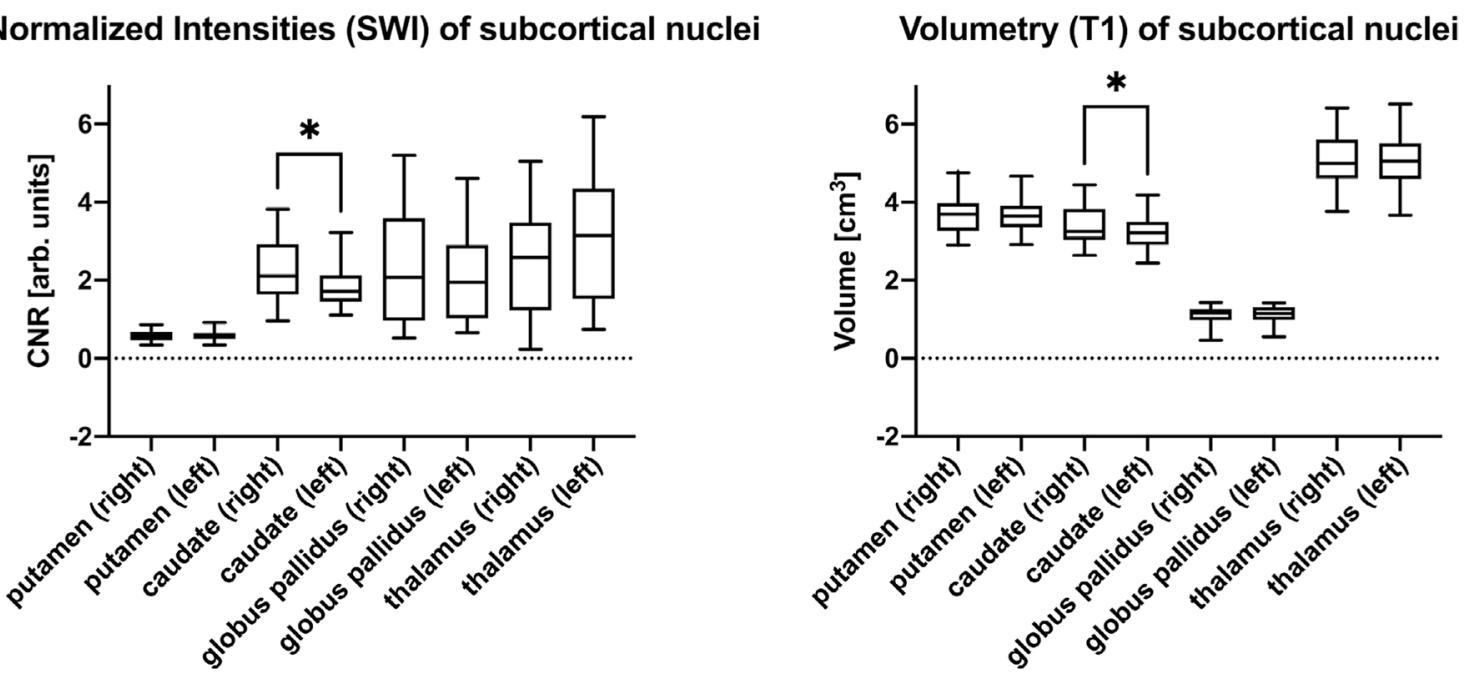

Fig. 2 Hemispherical side differences for ${ }^{31}$ P-MRSI measurements, normalized intensities (SWI), and volumetry (T1) of subcortical nuclei. Box plot diagrams are plotted with the median and the $95 \%$ confidence interval whiskers. ${ }^{* * * * * * * * *}$ : significance levels $\left({ }^{*}: \mathrm{P} \leq .05,{ }^{* *}: \mathrm{P} \leq .01,{ }^{* * *}\right.$ : $\mathrm{P} \leq .001$; ****:P $\leq .0001) .{ }^{31} \mathrm{P}-\mathrm{MRSI}:{ }^{31} \mathrm{Phosphorus}$ magnetic resonance spectroscopy imaging. arb. units arbitrary units. ATP adenosine triphosphate, CNR contrast-to-noise ratio, iP inorganic phosphate, $P C r$ phosphocreatinine, SWI susceptibility-weighted imaging

daily dose (LEDD) of $680 \pm 465 \mathrm{mg} / \mathrm{d} .57 \%(\mathrm{n}=17) \mathrm{PD}$ patients presented with left- and $43 \%(n=13)$ with rightlateralized symptoms.

\section{Hemispherical side differences in subcortical brain} bioenergetics, iron deposition, and volumetric measures. We observed that the ${ }^{31} \mathrm{P}$-MRSI-derived metabolite ratios were significantly lower in the right hemisphere (Fig. 2) inclusive of $(\beta \mathrm{ATP}+\mathrm{PCr}) / \mathrm{iP} \quad(\mathrm{t}(30)=5.58, \quad \mathrm{P}<0.0001)$ (right $5.9 \pm 2.6$ vs. left $9.9 \pm 3.3$ ), $\beta \mathrm{ATP} / \mathrm{iP}$ and $\mathrm{PCr} / \mathrm{iP}$ of the $(\beta \mathrm{ATP}+\mathrm{PCr}) / \mathrm{iP}$ ratio with significant side differences for $\beta$ ATP $/ \mathrm{iP}(\mathrm{t}(30)=6.96, \mathrm{P}<0.0001$, right: $1.7 \pm 0.6$, left:
$3.1 \pm 1.3)$ and $\mathrm{PCr} / \mathrm{iP}(\mathrm{t}(30)=4.07, \mathrm{P}<0.001$, right: $4.1 \pm 2.4$, left: $6.9 \pm 2.8$ ). Representative spectra to illustrate the hemispherical differences are highlighted in Additional file 1: Figure S1. The logistic regressions showed that lateralization of brain energy metabolite levels was not driven by the more severely affected body side of the study participants. The CNR values derived from the SWI images showed similar but less pronounced side differences for the caudate $(\mathrm{t}(30)=2.4, \mathrm{P}=0.025$, right: $3.4 \pm 0.5$, left: $3.2 \pm 0.5)$ but not for the other regions of interest. Volumetric measures revealed only significant side differences for the caudate $(\mathrm{t}(30)=2.32, \mathrm{P}=0.027$, right: $2.2 \pm 0.8$, left: $1.9 \pm 0.6)$. 
Table 1 Summary of multiple regression model results of $(\beta A T P+P C r) / i P$ vs. SWI CNR values

\begin{tabular}{|c|c|c|c|c|c|c|}
\hline Analysis of variance & SS & DF & MS & $\mathrm{F}$ & p-value & \\
\hline Regression & 48.79 & 4 & 12.20 & $F(4,57)=41.39$ & $\mathrm{P}<.0001^{* * * *}$ & \\
\hline SWI: putamen (CNR) & 15.46 & 1 & 15.46 & $F(1,57)=52.46$ & $P<.0001^{* * * *}$ & \\
\hline SWI: caudate (CNR) & 2.00 & 1 & 2.00 & $F(1,57)=6.78$ & $P=.0117^{*}$ & \\
\hline SWI: globus pallidus (CNR) & 2.98 & 1 & 2.98 & $F(1,57)=10.10$ & $P=.0024^{* *}$ & \\
\hline SWI: thalamus (CNR) & 0.79 & 1 & 0.79 & $F(1,57)=2.68$ & $P=.1074$ & \\
\hline Residual & 16.80 & 57 & 0.29 & & & \\
\hline Total & 65.58 & 61 & & & & \\
\hline Parameter Estimates & Variable & Estimate & SE & $95 \% \mathrm{Cl}$ & $\mathrm{T}$ & p-value \\
\hline$\beta 0$ & Intercept & 1.36 & 0.31 & $0.74 ; 1.98$ & 4.39 & $\mathrm{P}<.0001^{* * * *}$ \\
\hline$\beta 1$ & SWl: putamen (CNR) & 4.44 & 0.61 & $3.21 ; 5,67$ & 7.24 & $\mathrm{P}<.0001^{* * * *}$ \\
\hline$\beta 2$ & SWI: caudate (CNR) & 0.27 & 0.10 & $0.06 ; 0.48$ & 2.60 & $P=.0117^{*}$ \\
\hline$\beta 3$ & SWI: globus pallidus (CNR) & 0.19 & 0.06 & $0.07 ; 0,31$ & 3.18 & $\mathrm{P}=.0024^{* *}$ \\
\hline$\beta 4$ & SWI: thalamus (CNR) & 0.10 & 0.05 & $0.00 ; 0.18$ & 1.64 & $P=.1074$ \\
\hline \multicolumn{7}{|l|}{ Goodness of Fit } \\
\hline DF & 57 & & & & & \\
\hline Multiple R & 0.86 & & & & & \\
\hline$R^{2}$ & 0.74 & & & & & \\
\hline$R_{a d j}^{2}$ & 0.73 & & & & & \\
\hline SS & 0.74 & & & & & \\
\hline RMSE & 0.11 & & & & & \\
\hline Multicollinearity & Variable & VIF & $\begin{array}{l}\mathrm{R}^{2} \text { with other } \\
\text { variables }\end{array}$ & & & \\
\hline$\beta 0$ & Intercept & & & & & \\
\hline$\beta 1$ & SWl: putamen (CNR) & 1.59 & 0.37 & & & \\
\hline$\beta 2$ & SWI: caudate (CNR) & 1.18 & 0.15 & & & \\
\hline$\beta 3$ & SWI: globus pallidus (CNR) & 1.29 & 0.22 & & & \\
\hline$\beta 4$ & SWI: thalamus (CNR) & 1.21 & 0.17 & & & \\
\hline Normality of Residuals & Statistics & p-value & $\begin{array}{l}\text { Passed normality } \\
\text { test }(a=.05) ?\end{array}$ & & & \\
\hline Anderson and Darling (1952) & 0.58 & $P=0.13$ & Yes & & & \\
\hline D’Agostino et al. (1990) & 2.23 & $P=0.33$ & Yes & & & \\
\hline Shapiro and Wilk (1965) & 0.97 & $P=0.16$ & Yes & & & \\
\hline Massey (distance) (1951) & 0.08 & $P>.10$ & Yes & & & \\
\hline
\end{tabular}

The table summarizes the multiple regression model of ( $\beta A T P+P C r) / i P$ vs. SWI CNR values, including descriptive analyses. Apart from the overall significance test of the model, parameter estimates, goodness of fit, and necessary assumptions for multiple regression models were tested (absence of multicollinearity and normality of residuals). ${ }^{* * *} / * * * * * *$ : significance levels $\left(*: P \leq 0.05,{ }^{* *}: \mathrm{P} \leq 0.01,{ }^{* * *}: \mathrm{P} \leq 0.001 ;{ }^{* * * *}: \mathrm{P} \leq 0.0001\right)$

$C l$ confidence interval, CNR contrast-to-noise ratio, DF degrees of freedom, MS mean square, $R^{2}$ coefficient of determination, $R^{2}$ adj adjusted coefficient of determination, RMSE root mean square error, SE standard error, SS sum of squares, SWI susceptibility-weighted imaging, $T$ t-statistic, $F$ F-Statistic, VIF variance inflation factor

Subcortical brain iron deposition, but not the individual volumetric measurements, predicts the bioenergetic status of each hemisphere

Based on three metabolite ratios and two independent variable sets, we computed six multiple regression models. We observed a highly significant association for the model of $(\beta \mathrm{ATP}+\mathrm{PCr}) / \mathrm{iP}$ vs. the $\mathrm{CNR}$ values (putamen, caudate, globus pallidus, and thalamus) $(\mathrm{P}<0.0001)$ (see
Table 1). Here, the parameter estimates for the CNR of the putamen $(\mathrm{P}<0.0001)$, the caudate $(\mathrm{P}=0.0117)$, and globus pallidus $(\mathrm{P}=0.0024)$ significantly contributed to the prediction of our model. The overall goodness of fit resulted in a high adjusted coefficient of determination $R^{2}$ adj of 0.74 . The independent variables did not show a relevant degree of multicollinearity with variance inflation factors ranging from 1.18 to 1.59 and $R^{2}$ (among 
included variables) of only maximal 0.37 (putamen). Diagnostic tests for the normality of residuals were all passed (Table 1, Fig. 3: QQ plot). Furthermore, the present multiple linear regression model showed homoscedasticity (Fig. 3: Homoscedasticity plot), the residuals themselves were not predictive of the dependent variable (Fig. 3: Residual plot), and the selected parameters were not concerningly intertwined (Fig. 3: Parameter covariance matrix). For illustrative purposes, the second (uncorrected) significant $\left(\mathrm{P}=0.0393, R_{\text {adj }}^{2}=0.10\right)$ regression model $(\mathrm{PCr} / \mathrm{iP}$ vs. $\mathrm{CNR})$ is listed in the Supplementary Material (Additional file 1: Table S2, Figure S2). We could describe no significant findings or trends for the $\beta \mathrm{ATP} / \mathrm{iP}$ ratio vs. CNR regression model and the regression models with volumetric measures as independent variables.

\section{Neither subcortical brain iron deposition nor the individual volumetric measurements correlate with age, disease duration, or MDS-UPDRS-III}

Figure 4 summarizes the exploratory Pearson's correlations of our neuroimaging measures with demographic and clinical data. The logistic regressions with sex were negative, suggesting that sex did not confound on metabolite ratios and imaging findings. Neither SWI nor T1 imaging findings correlated with demographic or clinical data. Furthermore, we performed additional exploratory correlations due to the lack of a significant relationship with age, disease duration, and MDS-UPDRS-III.

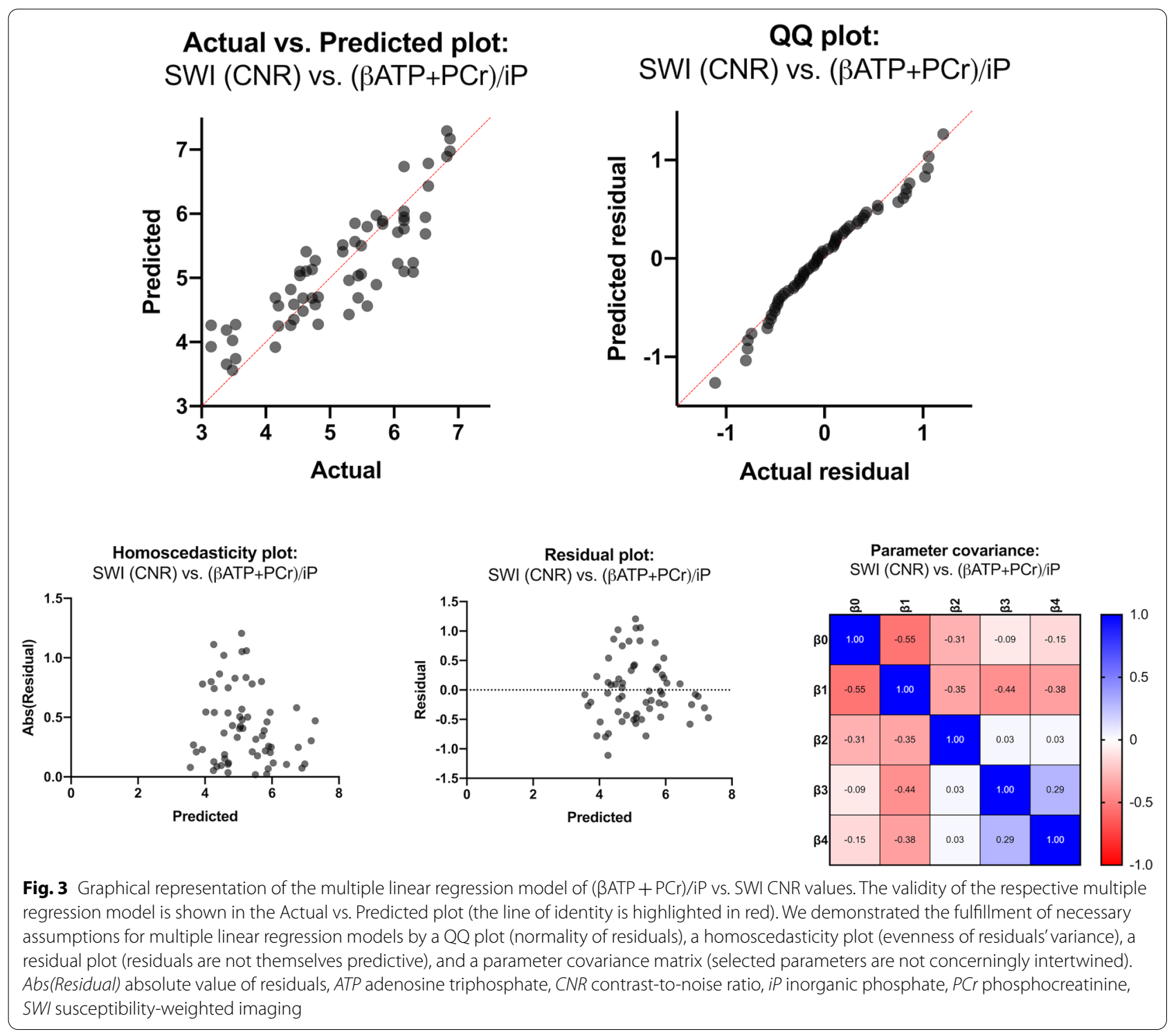




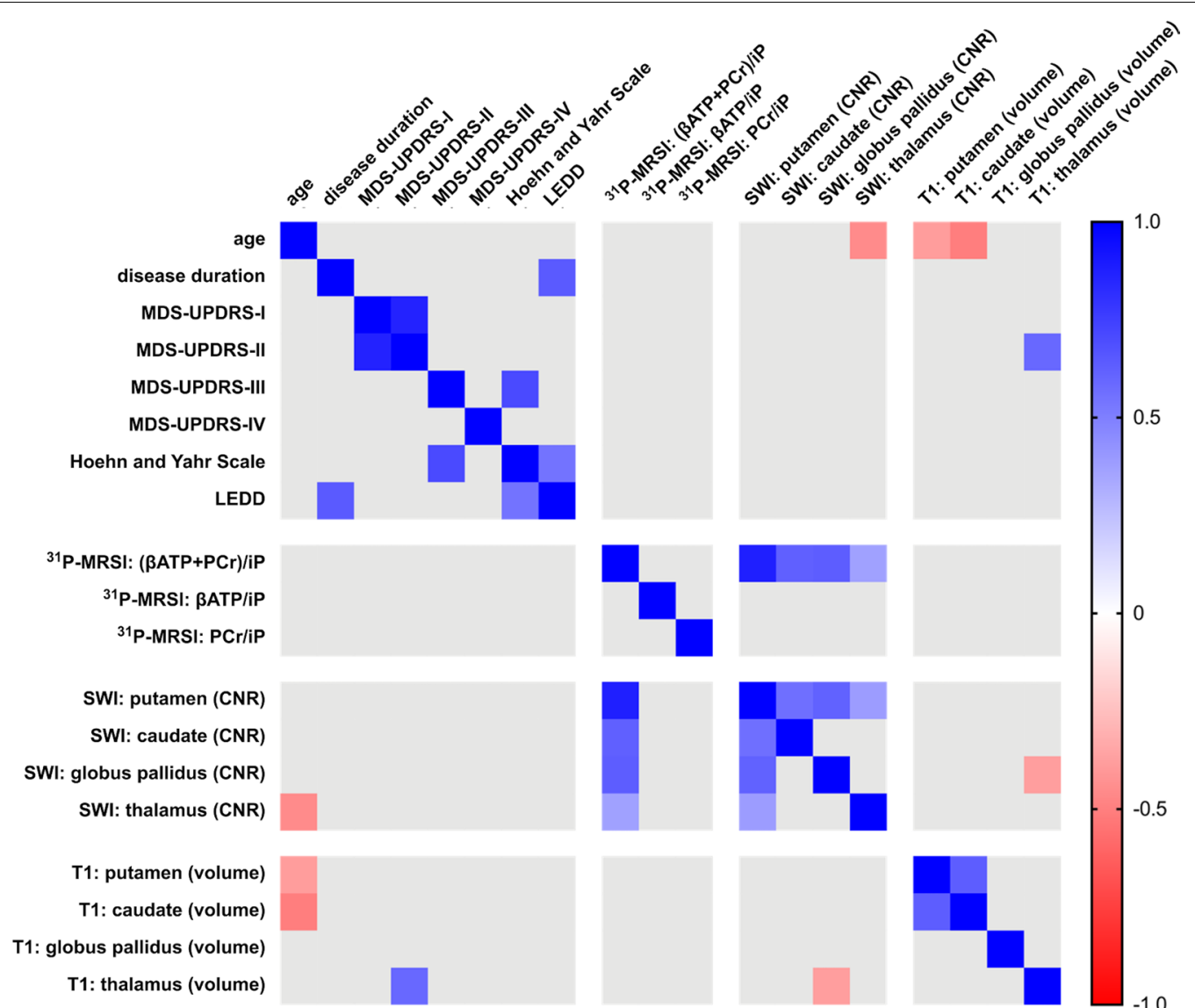

Fig. 4 Heatmap for the correlation analyses of demographic, clinical, and neuroimaging data. Presented are Pearson's correlation coefficients (thresholded with a p-value of $>.05$ ) as exploratory analyses (color-coded for negative and positive coefficients, see right scale). ${ }^{31} \mathrm{P}$-MRSI: ${ }^{31}$ Phosphorus magnetic resonance spectroscopy imaging. CNR contrast-to-noise ratio, LEDD levodopa equivalent daily dosage, MDS-UPDRS Movement Disorders Society Unified Parkinson's Disease Rating Scale, SWI susceptibility-weighted imaging

Interestingly, the SWI CNR values seemed to be of minor relevance to characterize the disease state with correlation coefficients ranging between \pm 0.40 . The same could be observed for the ${ }^{31} \mathrm{P}$-MRSI metabolite ratios.

\section{Discussion}

To the best of our knowledge, this study reports first the potential interconnectedness of bioenergetic disturbances and brain iron deposition level in patients with PD using in vivo neuroimaging. Here, subcortical brain iron deposition, particularly in the putamen and globus pallidus, was highly predictive of the overall amount of high-energy containing phosphates in our subjects. We observed no association with the individual volumetric measurements, highlighting the potential of ${ }^{31} \mathrm{P}-\mathrm{MRSI}$ and iron-weighted imaging as pathophysiology-orientated biomarkers. Our findings suggest that brain iron deposition is related to mitochondrial impairment in vivo. However, we could not determine a causal relationship between them. Future studies should address whether these findings might indicate therapeutic advancements to improve mitochondrial bioenergetics in patients by administering chelating agents.

The observed hemispherical differences in brain energy metabolism and iron distribution were unexpected findings. Previous reports suggest that the lateralization of distinct SWI findings is present in patients with PD or Multiple System Atrophy with predominant parkinsonism (MSA-P) (Hwang et al. 2015). In line with our findings, the putaminal tracer uptake of [(123)I] $\beta$-carboxymethyoxy-3- $\beta$-(4-iodophenyl)tropane PET indicated that the right hemisphere is predominantly affected in PD, being potentially related to handedness (Scherfler et al. 2012). Hemispheric side differences, in particular those concerning the role of the dominant hemisphere, could also be predictive for individual symptom presentation and disease progression (Ham et al. 2015; Riederer and Sian-Hülsmann 2012). Furthermore, the distribution of striatal dopamine content shows an asymmetric distribution in prodromal PD, being relevant 
for the subsequent motor symptom onset in patients with PD (Haaxma et al. 2010). Nevertheless, it remains elusive whether these previous findings would also result in bioenergetic alterations and should be considered in future multimodal imaging studies. In contrast, the absence of statistically significant correlations of our basal ganglia ${ }^{31} \mathrm{P}-\mathrm{MRSI}$ neuroimaging findings with the laterality of symptoms implies that dopaminergic dysfunction and bioenergetic depletion are not associated and likely represent independent pathophysiological traits.

However, the observed hemispherical differences yield important implications for future studies: ${ }^{31} \mathrm{P}$-MRSI studies often record a global signal (e.g., by using surface head coils), which might miss lateralized differences concerning an individual's brain anatomy (Rango et al. 2020). Frequent brain iron deposition in midbrain or brainstem structures of diseased individuals (such as in the substantia nigra of patients with PD) might also be a potential limitation, which could be assessed by iron-weighted neuroimaging (Péran et al. 2010; Yoshikawa et al. 2016; Guan et al. 2018). In contrast, ${ }^{31}$ P-MRSI-mediated examination of in vivo bioenergetics is substantially hampered in these brain structures by the relatively low spatial resolution and insufficient tissue homogeneity to yield satisfactory spectral quality for metabolite quantification. Interestingly, our neuroimaging measures were only marginally associated with the phenotype. In particular, the SWI measures are in contrast to previous reports where the iron deposition was related to disease duration or severity (Wang et al. 2016). This finding implicates the need for longitudinal studies that could address whether brain iron deposition is a consequence or rather a primary driver of neurodegeneration. The latter would be especially relevant as patients with increased brain iron deposition in early disease stages could benefit the most from targeted treatment strategies. Given the likely complexity of one individual's disease pathophysiology, it would be crucial to stratify patients by their outweighing etiology to stratify them and subsequently sustain clinical trial success in the future. The temporal dynamics of brain iron deposition and mitochondrial dysfunction are so far unknown. Subcortical iron deposition can be an epiphenomenon or an active driver of bioenergetic failure in patients with PD. The synthesis of Fe-Sulfur clusters is highly energy-dependent, and the depletion of ATP due to mitochondrial dysfunction can lead to an impaired assembly and accumulation of iron in mitochondria (Muñoz et al. 2016). Iron itself can promote the formation of reactive oxygen species, which additionally impacts mitochondrial homeostasis and can cause bioenergetic depletion (Muñoz et al. 2016). Most likely, these processes act as a vicious cycle (Muñoz et al. 2016). To better understand the temporal dynamics of brain iron deposition and mitochondrial dysfunction in the prolonged process of neurodegeneration, longitudinal studies would thus be necessary. Such studies would substantially benefit from the use of quantitative MRI methods, potentially improving the multi-site reliability of the upcoming findings (Barbosa et al. 2015; Weiskopf et al. 2013). As a limitation, SWI is also sensitive to compounds other than iron, e.g. calcium, potentially distorting the local magnetic field and generating image contrast (Liu et al. 2017). The combination of quantitative susceptibility imaging and relaxometry (e.g., by multiparameter mapping) might thus provide more information on the role of brain iron deposition in neurodegenerative disorders (Weiskopf et al. 2013). The combination of different iron-sensitive MRI methodologies might also lead to the specific detection of divalent and trivalent iron atoms in vivo, as preliminarily demonstrated in a phantom MRI study (Dietrich et al. 2017). Especially for the investigation of mitochondrial dysfunction in patients with PD, the role of divalent and trivalent iron in the production of reactive oxygen species might provide more detailed insights into the underlying biology of PD and could be used to map individual treatment responses to oxidative stress-targeted treatment regimes.

\section{Conclusions}

In this study, we demonstrated that subcortical brain iron deposition is highly predictive of mitochondrial impairment in patients with PD in vivo. Our findings highlight the interconnectedness of two important pathophysiological hallmarks of this disorder that were previously implicated by in-vitro and post-mortem experiments. Our preliminary experimental data support the potential use of chelating agents in individualized treatments for patients with PD. However, longitudinal studies are required to address the temporal aspects of the course of the disease and identify the window of opportunity for personalized therapies.

\section{Abbreviations}

PD: Parkinson's disease; SWI: Susceptibility-weighted imaging; LEDD: Levodopa equivalent daily dose; CNR: Contrast-to-noise ratio; FNIRT: FMRIB's Non-Linear Image Registration Tool; SD: Standard deviation; VOI: Voxel of interest; CSI: Chemical shift imaging; MSA-P: Multiple System Atrophy with predominant parkinsonism.

\section{Supplementary Information}

The online version contains supplementary material available at https://doi. org/10.1186/s10020-021-00426-9.

Additional file 1. Additional figures and tables. 


\section{Acknowledgements}

We thank Dr. Nathalie Schell and Dr. Alexander Balck for their support in patient recruitment.

\section{Authors' contributions}

JP designed the study, recruited study participants, performed the clinical and neuroimaging assessments, performed the neuroimaging and statistical analyses, and drafted the manuscript. MG established and improved the ${ }^{31}$ P-MRSI measurements, performed the neuroimaging analyses, and reviewed the manuscript. FG recruited study participants, performed the clinical and neuroimaging assessments, and reviewed the manuscript. SK recruited study participants, performed the clinical and neuroimaging assessments, and reviewed the manuscript. BE recruited study participants, performed the clinical and neuroimaging assessments, and reviewed the manuscript. MK designed the study, ensured study participant recruitment, and reviewed the manuscript. $\mathrm{HH}$ recruited study participants, performed the clinical and neuroimaging assessments, and reviewed the manuscript. CK designed the study, ensured study participant recruitment, and reviewed the manuscript. NB designed the study, ensured study participant recruitment, organized the study, wrote the first draft, and critically reviewed the manuscript. All authors read and approved the final manuscript.

\section{Funding}

JP received funding from the Parkinson's Foundation (Miami, Florida, USA), the Deutsche Parkinsongesellschaft (Berlin, Germany), and the Deutsche Forschungsgemeinschaft via the Clinician Scientist School Lübeck (DFGGEPRIS 413535489). NB received funding from the DFG (BR4328.2-1 (FOR2488), GRK1957) and the Collaborative Center for X-linked Dystonia-Parkinsonism.

\section{Availability of data and materials}

The datasets used and/or analyzed during the present study are available from the corresponding author on reasonable request.

\section{Declarations}

\section{Ethics approval and consent to participate}

This study has been approved by the ethics committee of the University of Lübeck (AZ 18_945). All participants gave written informed consent for participation.

\section{Consent for publication}

All enrolled study participants gave written informed consent for the publication of anonymized study-related data.

\section{Competing interests}

The authors declare that they have no competing interests.

\section{Author details}

'Institute of Neurogenetics, University of Lübeck, Ratzeburger Allee 160, 23538 Lübeck, Germany. ${ }^{2}$ Department of Neurology, University Medical Center Schleswig-Holstein, Campus Lübeck, Lübeck, Germany. ${ }^{3}$ Center for Brain, Behavior, and Metabolism, University of Lübeck, Lübeck, Germany. ${ }^{4}$ Department of Psychiatry and Psychotherapy, University Medical Center Schleswig-Holstein, Campus Lübeck, Lübeck, Germany.

Received: 22 September 2021 Accepted: 16 December 2021

Published online: 04 March 2022

\section{References}

Anderson TW, Darling DA. Asymptotic theory of certain "goodness of fit" criteria based on stochastic processes. Ann Math Statist. 1952;23:193-212.

Andersson JLR, Jenkinson M, Smith S. Non-linear registration aka spatial normalisation. FMRIB Technical Report TRO7JA2 2007.

Barbosa JHO, Santos AC, Tumas V, et al. Quantifying brain iron deposition in patients with Parkinson's disease using quantitative susceptibility mapping, R2 and R2. Magn Reson Imaging. 2015;33:559-65.

Chan S, Lian Q, Chen MP, et al. Deferiprone inhibits iron overload-induced tissue factor bearing endothelial microparticle generation by inhibition oxidative stress induced mitochondrial injury, and apoptosis. Toxicol Appl Pharmacol. 2018;338:148-58.

D'Agostino RB, Belanger A, D'Agostino RB. A suggestion for using powerful and informative tests of normality. Am Stat. 1990;44:316-21.

Devos D, Cabantchik Zl, Moreau C, et al. Conservative iron chelation for neurodegenerative diseases such as Parkinson's disease and amyotrophic lateral sclerosis. J Neural Transm (vienna). 2020;127:189-203.

Dietrich O, Levin J, Ahmadi SA, et al. MR imaging differentiation of Fe2+ and $\mathrm{Fe} 3+$ based on relaxation and magnetic susceptibility properties. Neuroradiology. 2017:59:403-9.

Gaki GS, Papavassiliou AG. Oxidative stress-induced signaling pathways implicated in the pathogenesis of Parkinson's disease. Neuro Mol Med. 2014;16:217-30.

Goetz CG, Tilley BC, Shaftman SR, et al. Movement Disorder Society-Sponsored Revision of the Unified Parkinson's disease Rating Scale (MDSUPDRS): Scale presentation and clinimetric testing results. Mov Disord. 2008;23:2129-70.

Guan XJ, Xie F, Xu XJ. The application value of quantitative susceptibility mapping in evaluating substantia nigra "swallow tail sign" in the diagnosis of Parkinson's disease. Chin J Contemp Neurol Neurosurg. 2018;18:247-51.

Haaxma CA, Helmich RC, Borm GF, Kappelle AC, Horstink MW, Bloem BR. Side of symptom onset affects motor dysfunction in Parkinson's disease. Neuroscience. 2010;170:1282-5.

Ham JH, Lee JJ, Kim JS, Lee PH, Sohn YH. Is dominant-side onset associated with a better motor compensation in Parkinson's disease? Mov Disord. 2015;30:1921-5.

Hwang I, Sohn CH, Kang KM, et al. Differentiation of Parkinsonism-predominant multiple system atrophy from idiopathic Parkinson disease using $3 T$ susceptibility-weighted MR imaging, focusing on putaminal change and lesion asymmetry. AJNR Am J Neuroradiol. 2015;36:2227-34.

Jenkinson M, Smith S. A global optimisation method for robust affine registration of brain images. Med Image Anal. 2001;5:143-56.

Jenkinson M, Bannister P, Brady M, Smith S. Improved optimization for the robust and accurate linear registration and motion correction of brain images. Neuroimage. 2002;17:825-41.

Jenkinson M. FMRIB Software Library (FSL). Internet. http://www.fmrib.ox.ac. uk/fsl; vol 23. Oxford: University of Oxford; 2008.

Jung WI, Staubert A, Widmaier S, et al. Phosphorus J-coupling constants of ATP in human brain. Magn Reson Med. 1997;37:802-4.

Kakhlon O, Breuer W, Munnich A, Cabantchik ZI. Iron redistribution as a therapeutic strategy for treating diseases of localized iron accumulation. Can J Physiol Pharmacol. 2010;88:187-96.

Lee DW, Kaur D, Chinta SJ, Rajagopalan S, Andersen JK. A disruption in ironsulfur center biogenesis via inhibition of mitochondrial dithiol glutaredoxin 2 may contribute to mitochondrial and cellular iron dysregulation in mammalian glutathione-depleted dopaminergic cells: implications for Parkinson's disease. Antioxid Redox Signal. 2009;11:2083-94.

Liang LP, Patel M. Iron-sulfur enzyme mediated mitochondrial superoxide toxicity in experimental Parkinson's disease. J Neurochem. 2004;90:1076-84.

Liu S, Buch S, Chen Y, et al. Susceptibility-weighted imaging: current status and future directions. NMR Biomed. 2017;30:1.

Massey FJ. The Kolmogorov-Smirnov test for goodness of fit. J Am Stat Assoc. 1951;46:68-78.

Mena NP, Bulteau AL, Salazar J, Hirsch EC, Núñez MT. Effect of mitochondrial complex I inhibition on Fe-S cluster protein activity. Biochem Biophys Res Commun. 2011;409:241-6.

Mochizuki H, Imai $\mathrm{H}$, Endo K, et al. Iron accumulation in the substantia nigra of 1-methyl-4-phenyl-1,2,3,6-tetrahydropyridine (MPTP)-induced hemiparkinsonian monkeys. Neurosci Lett. 1994;168(1-2):251-3.

Moos T, Morgan EH. The metabolism of neuronal iron and its pathogenic role in neurological disease review. Ann N Y Acad Sci. 2004;1012:14-26.

Muñoz Y, Carrasco CM, Campos JD, Aguirre P, Núñez MT. Parkinson's disease: the mitochondria-iron link. Parkinsons Dis. 2016;2016:7049108.

Park JS, Davis RL, Sue CM. Mitochondrial dysfunction in Parkinson's disease: new mechanistic insights and therapeutic perspectives. Curr Neurol Neurosci Rep. 2018;18:21.

Patenaude B, Smith SM, Kennedy DN, Jenkinson M. A Bayesian model of shape and appearance for subcortical brain segmentation. Neuroimage. 2011;56:907-22.

Péran P, Cherubini A, Assogna F, et al. Magnetic resonance imaging markers of Parkinson's disease nigrostriatal signature. Brain. 2010;133:3423-33. 
Postuma RB, Berg D, Stern M, et al. MDS clinical diagnostic criteria for Parkinson's disease. Mov Disord. 2015;30:1591-601.

Purvis L, Clarke WT, Biasiolli L, Rodgers VL. OXSA: an open-source magnetic resonance spectroscopy analysis toolbox in MATLAB. PLOS ONE. 2017;9:12.

Rango M, Dossi G, Squarcina L, Bonifati C. Brain mitochondrial impairment in early-onset Parkinson's disease with or without PINK1 mutation. Mov Disord. 2020;35:504-7.

Ren J, Sherry AD, Malloy CR. 31P-MRS of healthy human brain: ATP synthesis, metabolite concentrations, $\mathrm{pH}$, and $\mathrm{T} 1$ relaxation times. NMR Biomed. 2015;28:1455-62.

Riederer P, Sian-Hülsmann J. The significance of neuronal lateralisation in Parkinson's disease. J Neural Transm (vienna). 2012;119:953-62.

Schade S, Mollenhauer B, Trenkwalder C. Levodopa equivalent dose conversion factors: an updated proposal including opicapone and safinamide. Mov Disord Clin Pract. 2020;7:343-5.

Scherfler C, Seppi K, Mair KJ, et al. Left hemispheric predominance of nigrostriatal dysfunction in Parkinson's disease. Brain. 2012;135:3348-54.

Shapiro SS, Wilk MB. An analysis of variance test for normality (complete samples). Biometrika. 1965;52:591-611.

Sohn YS, Breuer W, Munnich A, Cabantchik ZI. Redistribution of accumulated cell iron: A modality of chelation with therapeutic implications. Blood. 2008;111:1690-9.

Timpka J, Cenci MA, Odin P. Etiology and pathogenesis of Parkinson's disease. Mov Disord Curricula. 2017;1:95-101.

Trufanov AG, Yurin AA, Buriak AB, Sandalov SA, Odinak MM, Litvinenko IV. Susceptibility-weighted MR imaging (SWI) of basal ganglia iron deposition in the early and advanced stages of Parkinson's disease. Nevrol Neiropsikhiatriya, Psikhosomatika. 2019;11:30-6.

Vanhamme L, van den Boogaart A, Van Huffel S. Improved method for accurate and efficient quantification of MRS data with use of prior knowledge. J Magn Reson. 1997;129:35-43.

Wang JY, Zhuang QQ, Zhu LB, et al. Meta-analysis of brain iron levels of Parkinson's disease patients determined by postmortem and MRI measurements. Sci Rep. 2016;6:36669.

Weiskopf N, Suckling J, Williams G, et al. Quantitative multi-parameter mapping of R1, PD*, MT, and R2* at 3T: A multi-center validation. Front Neurosci. 2013;7:95.

Yoshikawa K, Tanaka M, Shimidzu Y, Kitamura N. Depletion of nigrosome 1 in parkinsonian brains as demonstrated by the loss of the "swallow tail sign" on 3t MRI-SWI. Neurology. 2016;86:1.

Zhang J, Zhang Y, Wang J, et al. Characterizing iron deposition in Parkinson's disease using susceptibility-weighted imaging: an in vivo MR study. Brain Res. 2010;1330:124-30.

\section{Publisher's Note}

Springer Nature remains neutral with regard to jurisdictional claims in published maps and institutional affiliations.

Ready to submit your research? Choose BMC and benefit from:

- fast, convenient online submission

- thorough peer review by experienced researchers in your field

- rapid publication on acceptance

- support for research data, including large and complex data types

- gold Open Access which fosters wider collaboration and increased citations

- maximum visibility for your research: over $100 \mathrm{M}$ website views per year

At BMC, research is always in progress.

Learn more biomedcentral.com/submissions 